\title{
ACCURATELY CALCULATING THE SOLAR ORIENTATION OF THE TIANGONG-2 ULTRAVIOLET FORWARD SPECTROMETER
}

\author{
LIU Zhiwen $^{1}{ }^{*}$, Li Shengyang ${ }^{1}$ \\ ${ }^{1}$ Key Laboratory of Space Utilization, Technology and Engineering Center for space Utilization, Chinese Academy of Sciences. \\ Beijing, 100094, China-(zwliu, shyli)@csu.ac.cn
}

KEY WORDS: TianGong-2, Ultraviolet Forward Spectrometer, Solar Orientation, Azimuth Angle, Attitude Angle, Coordinate System

\begin{abstract}
:
The Ultraviolet Forward Spectrometer is a new type of spectrometer for monitoring the vertical distribution of atmospheric trace gases in the global middle atmosphere. It is on the TianGong-2 space laboratory, which was launched on 15 September 2016. The spectrometer uses a solar calibration mode to modify its irradiance. Accurately calculating the solar orientation is a prerequisite of spectral calibration for the Ultraviolet Forward Spectrometer. In this paper, a method of calculating the solar orientation is proposed according to the imaging geometric characteristics of the spectrometer. Firstly, the solar orientation in the horizontal rectangular coordinate system is calculated based on the solar declination angle algorithm proposed by Bourges and the solar hour angle algorithm proposed by Lamm. Then, the solar orientation in the sensor coordinate system is achieved through several coordinate system transforms. Finally, we calculate the solar orientation in the sensor coordinate system and evaluate its calculation accuracy using actual orbital data of TianGong-2. The results show that the accuracy is close to the simulation method with STK (Satellite Tool Kit), and the error is not more than $2 \%$. The algorithm we present does not need a lot of astronomical knowledge, but only needs some observation parameters provided by TianGong- 2 .
\end{abstract}

\section{INTRODUCTION}

The Ultraviolet Forward Spectrometer is a new type of spectrometer for comprehensively detection the near-surface ultraviolet radiation characteristics of the global middle atmosphere with fine spectral resolution. It is on the TianGong2 space laboratory, which was launched on 15 September 2016. The spectral range of the spectrometer includes UV-visible-near infrared. The spectrometer can sliced detect of the earth surface atmosphere, which can reflect vertical distribution of trace gases. The spatial and temporal distribution of multi-directional atmospheric trace gases can be obtained by the inversion calculation, which can serve the atmospheric environment monitoring and atmospheric science research. It provides a new data source for exploration atmosphere environment.

The Ultraviolet Forward Spectrometer adopts solar calibration mode to modify the spectral irradiance, the calibration source is the sun. Accurately calculating the solar orientation in the sensor coordinate system is a prerequisite of spectral calibration for the spectrometer. At present, Blanco-Muriel M (2001), LU W H (2008) and MOSKWA J J (1992) were calculated solar orientation in the field of ground tracking, but also in the field of remote sensing applications by Zhang Q(2011). The calculation method mostly adopt Michalsky's theoretical expansion method recommended by the World Meteorological Organization, or Wang B Z(2008)'s data simulation method. However, Du C X (2010a), MEEUS J (1998) and COOPER P I (1969)have shown that a great deal of astronomical knowledge is often required in calculating the solar orientation. Although its accuracy is a little higher than others, it is not fit for engineering applications.
In this paper, a method of calculating the solar orientation is proposed according to imaging geometric characteristics of the spectrometer.

(1).Firstly, several coordinate systems are introduced, such as, Earth Centred Earth Fixed Coordinate System(ECEF), Orbital Coordinate System(ORB), Body Coordinate System(BODY), Sensor Coordinate System(SCS), and so on.

(2).Secondly, the sub-satellite point geographic coordinates is calculated.

(3).Thirdly, the solar orientation of the sub-satellite point in the horizontal rectangular coordinate system is calculated, based on the solar declination angle algorithm proposed by Bourges and the solar hour angle algorithm proposed by Lamm.

(4).finally, the solar orientation in the sensor coordinate system is calculated, by several coordinate system transform from the horizontal rectangular coordinate system to the sensor coordinate system.

\section{RELATED COORDINATE SYSTEMS}

1. Earth Centered Earth Fixed Coordinate System(ECEF): a fixed earth coordinate system whose origin is at the mass center of the Earth. The $\mathrm{Z}_{\mathrm{ECEF}}$ axis points from the origin to the mean north pole. The $\mathrm{X}_{\mathrm{ECEF}}$ axis points from the origin to the intersection of the prime meridian and the equator.

\footnotetext{
* Corresponding author
} 
The $\mathrm{Y}_{\mathrm{ECEF}}$ axis is cross product of the $\mathrm{Z}_{\mathrm{ECEF}}$ axis and the $\mathrm{X}_{\mathrm{ECEF}}$ axis.

2. Orbital Coordinate System(ORB): the origin is at the mass center of TianGong-2. The $\mathrm{Z}_{\mathrm{ORB}}$ axis points from the origin to the mass center of the Earth. The $Y_{\mathrm{ORB}}$ axis is cross product of the $Z_{\mathrm{ORB}}$ axis and the instantaneous speed of TianGong-2. The $\mathrm{X}_{\mathrm{ORB}}$ axis is the cross product of the $\mathrm{Y}_{\mathrm{ORB}}$ axis and the $\mathrm{Z}_{\mathrm{ORB}}$ axis.

3. Body Coordinate System(BODY): the origin is in the mass center of TianGong-2. The $\mathrm{X}_{\mathrm{BODY}}, \mathrm{Y}_{\mathrm{BODY}}$, and $\mathrm{Z}_{\mathrm{BODY}}$ take the three main inertia axes of TianGong-2. The $X_{B O D Y}$ is along the horizontal axis of TianGong-2, the $\mathrm{Y}_{\mathrm{BODY}}$ is along the longitudinal axis towards TianGong- 2 and the $Z_{B O D Y}$ is determined according to the right-hand rule.

4. Sensor Coordinate System(SCS): the origin is at the projection center of the spectrometer. The $Z_{S C S}$ axis is from the origin to the main optical axis, the $\mathrm{Y}_{\mathrm{SCS}}$ axis is parallel to the $\mathrm{X}_{\mathrm{BODY}}$ axis of the body coordinate system, and the $\mathrm{X}_{\mathrm{SCS}}$ axis is the cross product of the $\mathrm{Y}_{\mathrm{SCS}}$ axis and the $\mathrm{Z}_{\mathrm{SCS}}$ axis.

5. Horizontal Rectangular Coordinate System(HCS ) : taking a point on the ground as the origin, the origin normal is the $\mathrm{Z}_{\mathrm{HCS}}$ axis (pointing to the zenith is positive), the meridian direction is the $X_{\mathrm{HCS}}$ axis, the $\mathrm{Y}_{\mathrm{HCS}}$ axis is the cross product of the $\mathrm{X}_{\mathrm{HCS}}$ and the $\mathrm{Z}_{\mathrm{HCS}}$ axis.

\section{SOLAR ORIENTATION CALCULATING}

\subsection{Sub-satellite Point Coordinates}

A sub-satellite point is vertical projection of the satellite on the earth's surface, usually expressed with geographic latitude and longitude.

The geographical latitude formula is:

$$
\sin (\phi-\delta)=\frac{R_{E}}{r}\left[f \sin (2 \delta)+f^{2} \sin (4 \delta)\left(\frac{R_{E}}{r}-\frac{1}{4}\right)\right]
$$

The longitude formula is:

$$
\lambda=\alpha-\alpha_{G}
$$

Where, $R_{E}$ is the equatorial radius of the earth, $(r, \delta, \alpha)$ is the platform spherical coordinates in the earth centred inertial Coordinate System, $a_{G}$ is the Greenwich ascension.

\subsection{Solar Orientation of Sub-satellite Points}

The solar orientation in the horizontal rectangular coordinate system includes the solar elevation angle and the solar azimuth.

1. the solar elevation angle $\sinh _{\Theta}=\sin \delta \sin \phi+\cos \delta \cos \phi \cos \tau$

2. the solar azimuth

$$
\sin A=\left(\sinh _{\Theta} \sin \phi-\sin \delta\right) /\left(\cosh _{\Theta} \cos \phi\right)
$$

Where, $\mathrm{h}_{\Theta}$ is the solar elevation angle, $A$ is the solar azimuth, $\delta$ is the solar declination Angle, $\tau$ is the solar hour angle, $\phi$ is the local latitude.

3.2.1 Solar Declination Angle( $\delta$ ): The solar declination angle is between the plane of the earth's equator, and the connection between the center of the earth and the center of the sun. It is an amount that approximates the annual change, and its value varies from approximately $-23.45^{\circ}$ to $23.45^{\circ}$. Due to the complex motion laws of the earth around the sun, in order to obtain the true value of the solar declination angle, highaccuracy real-time astronomical observations or table lookups from the astronomical almanac are needed, which is not fit for on-orbit solar calibration observations. The four typical simple calculation methods of the solar declination angle mainly include COOPER P I(1969), IQBAL M (1983), STNE W B (1985) and Bourges (1985). Among them, Bourges (1985) proposed an algorithm for calculating the declination angle in 1985. The maximum calculation error of the method is $0.025^{\circ}$ which is shown by Du C X (2010b). It can meet the needs of some engineering applications. This paper uses the algorithm to calculate the solar declination angle.

The formula of calculating the solar orientation is:

$$
\delta=0.3723+23.2567 \sin (w t)+0.1149 \sin (2 w t)-0.1712 \sin (3 w t)
$$$$
-0.7580 \cos (w t)+0.3656 \cos (2 w t)+0.0201 \cos (3 w t)
$$

where, $w=\frac{360}{365.2422}, t=n-1-n_{0}, n$ is the num of days in a year, $n_{0}=78.801+[0.2422($ year -1969$)]-I N T[0.25($ year -1969$)]$.

3.2.2 Solar Hour Angle $(\tau)$ : The solar angle is the dihedral angle between the solar time circle and the observer's meridian circle. Similar to the solar declination angle, the solar's time angle changes every moment, and it can usually only be estimated using some approximate formulas with different precisions. Several types of typical simple algorithms for calculating the solar time angle include IQBAL M (1983), DUFFIE J A (1980) and Lamm (1981) [10,11,14,15]. Among them, this paper uses the algorithm proposed by Lamm (1981)

$$
\tau(n)=\sum_{k=0}^{5}\left[A_{k} \cos \left(\frac{2 \pi \mathrm{k} n}{365.25}\right)+\mathrm{B}_{k} \sin \left(\frac{2 \pi \mathrm{k} n}{365.25}\right)\right]
$$

where, $n$ is the number of days to cycle from 1 to 4 years from each leap year to 1,461 days on the last day.

\begin{tabular}{ccc}
\hline $\mathrm{k}$ & $\mathrm{A}_{\mathrm{k}} / \mathrm{h}$ & $\mathrm{B}_{\mathrm{k}} / \mathrm{h}$ \\
0 & $2.0870 \mathrm{e}^{-4}$ & 0 \\
1 & $9.2869 \mathrm{e}^{-3}$ & $-1.2229 \mathrm{e}^{-1}$ \\
2 & $-5.2258 \mathrm{e}^{-2}$ & $-1.5698 \mathrm{e}^{-1}$ \\
3 & $-1.3077 \mathrm{e}^{-3}$ & $-5.1602 \mathrm{e}^{-3}$ \\
4 & $-2.1867 \mathrm{e}^{-3}$ & $-2.9823 \mathrm{e}^{-3}$ \\
5 & $-1.5100 \mathrm{e}^{-4}$ & $-2.3463 \mathrm{e}^{-4}$ \\
\hline
\end{tabular}

Table 1 . The value of the parameter $A_{k}$ and $B_{k}$ 


\subsection{Coordinate Systems Transform}

The solar orientation in the sensor coordinate system is achieved by transforming the solar orientation in the horizontal rectangular coordinate system to the sensor coordinate system.

3.3.1 Solar Orientation Vector $S_{1}$ : It represents a solar orientation vector in the horizontal rectangular coordinate system.

$$
\mathrm{S}_{1}=\left[\begin{array}{c}
\cos \left(\mathrm{h}_{\Theta}\right) \cos (A) \\
\cos \left(\mathrm{h}_{\Theta}\right) \sin (A) \\
\sin \left(\mathrm{h}_{\Theta}\right)
\end{array}\right]
$$

3.3.2 Conversion Matrix $T_{\text {HCStoECEF }}:$ It represents a conversion matrix from the horizontal rectangular coordinate system to the earth fixed coordinate system.

$$
T_{H C S t o E C E F}=R_{x}\left(90^{\circ}-\lambda\right) \mathrm{R}_{\mathrm{z}}\left(90^{\circ}+\phi\right)
$$

Where,

$$
\begin{array}{r}
R_{x}\left(90^{\circ}-\lambda\right)=\left(\begin{array}{ccc}
1 & 0 & 0 \\
0 & \cos \left(90^{\circ}-\lambda\right) & \sin \left(90^{\circ}-\lambda\right) \\
0 & -\sin \left(90^{\circ}-\lambda\right) & \cos \left(90^{\circ}-\lambda\right)
\end{array}\right) \\
R_{z}\left(90^{\circ}+\phi\right)=\left(\begin{array}{ccc}
\cos \left(90^{\circ}+\phi\right) & \sin \left(90^{\circ}+\phi\right) & 0 \\
-\sin \left(90^{\circ}+\varphi\right) & \cos \left(90^{\circ}+\phi\right) & 0 \\
0 & 0 & 1
\end{array}\right)
\end{array}
$$

3.3.3 Conversion Matrix $T_{E C E F t o O R B}:$ It represents a conversion matrix from the earth fixed coordinate system to the orbital coordinate system.

$$
T_{\text {ECEFtoORB }}=\left[\begin{array}{lll}
b_{1} & b_{2} & b_{3}
\end{array}\right]
$$

Where, $b_{3}=-P /\|P\|, b_{2}=\left(b_{3} \times V\right) /\left\|b_{3} \times V\right\|, b_{1}=b_{2} \times b_{3}, P$ is position vectors of TianGong-2, $V$ is platform speed vectors of TianGong-2.

\subsubsection{Conversion Matrix $T_{O R B t O B O D Y}:$ It represents a} conversion matrix from the orbital coordinate system to the body coordinate system.

$$
T_{\text {ORBtoBODY }}=T_{y} \times T_{\mathrm{x}} \times T_{z}
$$

Where,

$$
\begin{aligned}
& T_{z}=\left[\begin{array}{ccc}
\cos (\psi) & \sin (\psi) & 0 \\
-\sin (\psi) & \cos (\psi) & 0 \\
0 & 0 & 1
\end{array}\right] \\
& T_{x}=\left[\begin{array}{ccc}
1 & 0 & 0 \\
0 & \cos (\varphi) & \sin (\varphi) \\
0 & -\sin (\varphi) & \cos (\varphi)
\end{array}\right], \\
& T_{y}=\left[\begin{array}{ccc}
\cos (\theta) & 0 & \sin (\theta) \\
0 & 1 & 0 \\
-\sin (\theta) & 0 & \cos (\theta)
\end{array}\right],
\end{aligned}
$$

and the $\varphi, \theta, \psi$ is roll angles, pitch angles and yaw angles of TianGong-2, respectively.

3.3.5 Conversion Matrix $T_{B O D Y t o C S C}:$ It represents a conversion matrix from the body coordinate system to the sensor coordinate system.

$$
T_{\text {BODYtoCSC }}=\left[\begin{array}{ccc}
1 & 0 & 0 \\
0 & \cos (\alpha) & \sin (\alpha) \\
0 & -\sin (\alpha) & \cos (\alpha)
\end{array}\right]
$$

Where, ${ }^{\alpha}$ is the sensor installation angle in the sensor coordinate system.

3.4 Solar Orientation Vector $S_{2}$ : It represents a solar orientation vector in the sensor coordinate system. The formula is :

$$
\mathrm{S}_{2}=\mathrm{T}_{\text {BODYtoCSC }} \times \mathrm{T}_{\text {ORBtoBODY }} \times \mathrm{T}_{\text {ECEFtoORB }} \times \mathrm{T}_{H C S t o E C E F} \times S_{1}
$$

\section{EXPERIMENTS AND ANALYSIS}

Finally, we calculate the solar orientation in the sensor coordinate system using the actual related data of TianGong-2, and evaluate its accuracy.

\subsection{Calculation results}

Figure 1 shows the curves of the angle between the solar orientation vector and the Y-axis of the sensor coordinate system during a period of time when the Ultraviolet Forward Spectrometer directly observes the sun.

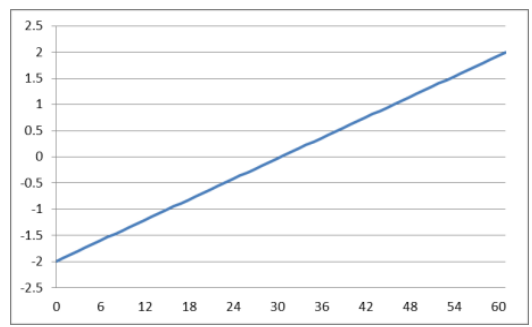

Figure 1. The angle between the solar orientation vector and the $\mathrm{Y}$ axis of the sensor coordinate system during a period of time

Figure 2 shows the curves of the angle between the solar orientation vector and the $\mathrm{X}$-axis in the sensor coordinate system for a period of time when the ultraviolet forward spectrometer directly observes the sun.



Figure 2. The angle between the solar orientation vector and the $\mathrm{X}$ axis of the sensor coordinate system during a period of time

\subsection{Accuracy verification}

The solar orientations are calculated respectively by the algorithm proposed in this paper and the simulation algorithm using the STK commercial software, and compared the differences between them.

The blue curve is the algorithm proposed in this paper, The red curve is the STK simulation algorithm. 


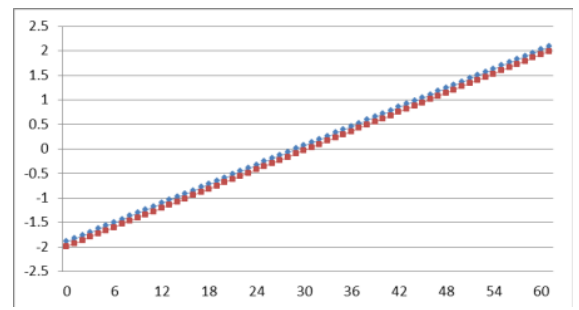

Figure 1. The angle between solar orientation vector and the $\mathrm{Y}$ axis of the sensor coordinate system

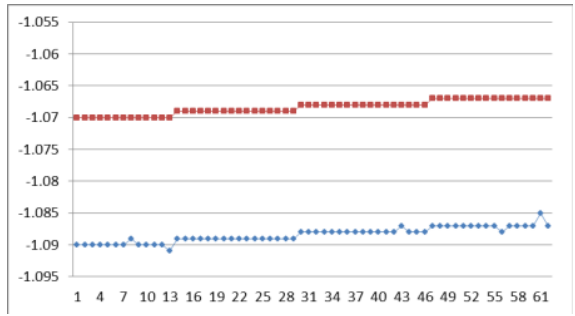

Figure 2. The angle between solar orientation vector and the $X$ axis of the sensor coordinate system during a period of time

The results show that the algorithm is close to the accuracy of the simulation algorithm using the STK commercial software, and the error is not more than $2 \%$. So the correctness of the algorithm is further verified. And The algorithm we present does not need astronomical knowledge, and only uses the observation parameters of TianGong- 2 .

\section{ACKNOWLEDGEMENTS (OPTIONAL)}

Thanks to China Manned Space Engineering for providing space science and application data products.

\section{CONCLUSIONS}

The solar orientation calculation method proposed in this paper is mainly based imaging geometrical relations of TianGong- 2 . And the accuracy is close to the simulation algorithm of STK commercial software. So, the method proposed in this paper can meet the demand of on-orbit solar calibration. And, it's not need any astronomy knowledge in calculation process, which is fit to some engineering applications. It has certain reference significance in the field of remote sensing data preprocessing for calculating the solar orientation.

\section{REFERENCES}

Blanco-Muriel M, Alarcón-Padilla D C, López-Moratalla T, et al. 2001.Computing the solar vector. Solar Energy, 70(5):431441.

Lv W H, He X L, Yu H J.2008.Development of full-automatic solar tracker and its applications. Optics and Precision Engineering ,16(12): 2544-2550.

MOSKWA J J,HEDRICK J K. 1992. Modeling and validation of automotive engines for control algortthm development. Transactions of the ASME,114(2): 278.

Zhang Q, Ye X, Fang W.2011.Calculation of sun's location on the sun synchronous orbit satellite and its precision analysis.Journal of the Graduate School of the Chinese Academy of Sciences, 28(3): 310-314.

Wang B Z,Tang J. 2001. Comparison of the different methods for solar position calculation. Acta Energiae Solaris Sinica,22(4):413-417.
MEEUS J. 1998. Astromomical algorithms. USA: WillmannBell Inc, pp.157-159.

Du C X, Wang P, Ma C F.2010a.Algorithm for the calculation of solar position by using astronomical almanac. RENEWABLE ENERGY RESIYRCES, 28(3): 85-92.

COOPER P I. 1969. The absorption of radiation in solar stills[J].Solar Energy, 12(3):333-346.

IQBAL M. 1983.An Introduction to Solar Radiation. New York. Academic Press

STNE W B. 1985.Solar Energy Fundamentals and Design With Computer Applications. New York. Jane Wiley \& Sons, BOURGES B. 1985. Improvement in solar declination computation. Solar Energy,35(4):367-369.

Du C X, Wang P, Ma C F.2010b. A high accuracy algorithm for the calculation of solar position. ENERGY ENGINEERING, (2): 41-44.

DUFFIE J A. 1980 Solar Engineering of Thermal Processes. New York.Jane Wiley \& Sons,

LAMM L O . 1981. A new analytic expression for the equation of time. Solar Energy, 26(5):465.

Revised March 2018 